\title{
NodeMCU and IoT-based Safety and Security Ecosystem for Heavy Vehicles
}

\author{
Ashish Patwari $^{1}$, P. Sai Sri Bhavya ${ }^{2}$, R.K. Maheswari ${ }^{3}$ \\ ${ }^{1}$ School of Electronics Engineering, Vellore Institute of Technology, Vellore, Tamil Nadu, India. \\ *Corresponding Author: ashish.p@vit.ac.in \\ 2 psaisribhavya@gmail.com \\ 3 maheswari6543@gmail.com
}

\begin{abstract}
This paper explains the design and implementation of a safety system for heavy vehicles. A small-scale embedded system based on Node MicroController Unit (NodeMCU) is proposed and prototyped here. Peripherals such as Global Positioning System (GPS) receiver (for location updates), tilt sensor (for accident detection), gas sensor (for alcohol activity detection), RFID tags and reader (to monitor entry and exit of vehicles in restricted areas), a touch sensor (to indicate an emergency) etc., have been used in this system. A major improvement over existing accident alert systems is that the proposed design makes use of ultrasonic sensors to indicate over-height of the vehicles approaching underpasses (tunnels or rail over road bridges) on highways. A sound alarm is triggered if the vehicle's height is more than the entry point of the underpass so that the driver can slow down the vehicle. A working prototype of the overall system has been implemented and tested successfully. The data gathered by all sensors is sent to the cloud using the Message Queuing Telemetry Transport (MQTT) protocol and the user can monitor all the updates using his/her android smartphone.
\end{abstract}

Key words: Accident Detection, Internet of Things (IoT), Node MCU, Over-height indication, Radio Frequency Identification (RFID), Ultrasonic sensor, Vehicle safety system.

\section{INTRODUCTION}

The high demand for transportation of goods in heavy vehicles has increased the traffic hazards. Life of people is under high risk due to road accidents. In the year 2018 alone, as per the official statistics, there were nearly 5 lakh (500 thousand) accidents and one lakh fifty thousand (150 thousand) deaths in India. Around 10\% of the dead were lorry and truck drivers. Overloaded vehicles caused around $12 \%$ of fatalities. Worst part of this was that almost $50 \%$ of the total deaths involved youth between $18-35$ years of age [1], [2]. The main reasons behind such accidents were: over speeding, non-use of helmets and seat belts, driving on the wrong side of the road, overloaded vehicles, bad roads, cellphone driving and drunken driving. Two-wheelers accounted for one third of the total deaths.

Passenger buses and luxury cars often come with lots of safety features embedded into them. A range of sensors, electronic control units (ECUs), communication protocols and rigorous testing go into the design of such vehicles. On the other hand, trucks and lorries which play a vital role in logistic services do not enjoy such on-board navigational and safety features. The utility of lorries and trucks in the transportation of goods is undisputed. They offer the much-needed last mile connectivity and act as a backbone to the entire supply chain system. Auto-rickshaws too play a major role in carrying commuters to their destinations. They are widely found in urban, sub-urban and rural areas of Asia, Africa and South America. In spite of the usefulness of such vehicles, they are not privileged enough to have on-board electronic safety systems often found in luxury buses and cars (at least not in present-day vehicles operating in India). Such automobiles are highly vulnerable to accidents and there are no mechanisms to report the occurrence of an accident or its location.

The specific improvements proposed in this paper that differentiate it from existing vehicle safety systems are as follows: -

- Radio Frequency Identification (RFID): The vehicle is equipped with an RFID tag. Using a suitable RFID reader at the entry and exit points of factories, the authorities can keep track of the movement of trucks inside the factory premises, the number of trips completed by a truck on a given day, the time of entry and exit of trucks etc. Also, RFID technology could help in granting or denying access to vehicles in restricted zones based on whether or not the tags are recognized by the reader.

- Over-height Indication: An ultrasonic sensor detects (from a distance) whether the underpass can contain 
the vehicle's height. If the vehicle is taller than the underpass's entry point, a sound buzzer is activated well-ahead of the entry point so that the driver can slow down the vehicle.

- Use of NodeMCU: The proposed system makes use of NodeMCU instead of powerful processors such as Raspberry Pi or ARM. The main reason for this is that the proposed design mainly relies on decision making and does not involve any complex computational tasks. Additionally, NodeMCU is quite inexpensive and simple than the other two. In particular, NodeMCU has lesser pins and consumes less power than Raspberry Pi.

Other regular features such as monitoring the vehicle's location, accident detection, accident reporting, alcohol activity detection etc., are included in the design. However, the proposed system does not deal with drowsiness detection.

The rest of the paper is organized as follows. Section 2 gives an overview of existing methods. Section 3 explains the proposed method. Section 4 presents the methodology followed to design and test the prototype. Section 5 presents the results obtained from the prototype. Section 6 provides a few future directions and concludes the paper.

\section{EXISTING METHODS}

Many vehicle safety systems have been proposed and prototyped in the recent past. An IoT-based system which processes data gathered from mobile sensors to monitor vehicular traffic and for accident prevention has been proposed recently [3]. A car safety system using ARM7 microcontroller, GPS and GSM has been proposed in [4]. The above system uses a tilt sensor to detect the occurrence of accidents and MQ-135 vapor sensor to detect alcohol activity.

An IoT-based system to detect drunken driving using the 8-bit STC12C5A16AD single chip microcontroller was proposed in [5]. The system uses four MQ-3 sensors to detect alcohol activity, disables the vehicle ignition and sends location alerts to the caretakers of the driver. A similar system based on Raspberry Pi, heart rate sensor, camera and alcohol sensor was proposed in [6]. A fleet monitoring system which can provide on-demand location updates and drunken driving alerts to the owners of fleet services was designed and demonstrated in [7]. However, the system had no mechanism for accident detection. An improved version of the above system with accident alert mechanism was presented in [8].

An Arduino-based accident alert system which can send Short Messaging Service (SMS) messages to ambulance helplines with an auto toll pay mechanism to reduce the waiting time at toll gates was proposed in [9]. A safety system for vehicles based on virtual traffic management has been proposed in [10]. The current location of the vehicle is obtained using GPS and the existing road rules in that particular area are informed to the driver. The system also has the ability to impose the rules in case the driver does not abide by the rules.

More recently, a system that can read the expression of driver and monitor alcohol activity has been proposed in [11]. In another prototype [12], a combination of Arduino and Raspberry Pi has been used to control vehicle ignition using multi-factor authentication and to monitor vital parameters such as fuel levels, engine temperature, proximity of obstacles etc. The vehicle ignition starts only after successful outcomes from face recognition, fingerprint identification and alcohol sensing modules. A vehicle security and accident alert system has been proposed in [13]. It consists of an anti-theft system for vehicles where fingerprint, password and one-time password (OTP) authentication are used to start the vehicle. The design also has a feature of geo fencing and accident detection. An ATmega328 based system which uses MQ-303A sensor for monitoring alcohol activity among drivers was recently proposed in [14]. It has to be noted that the MQ-303A sensor is tailor-made for alcohol detection whereas other sensors such as MQ-2 (gas/smoke sensing), MQ-5 (natural gas, liquified petroleum gas (LPG) sensing) and MQ-135 (air quality sensing) have more generic functionality.

Apart from drunken driving, drowsiness of drivers has also been the main cause for a number of accidents. Authors in [15] have excellently reviewed the existing literature to enlist the methods which are currently in vogue for detecting drunken driving. The measures to detect drowsy drivers can be largely classified into three types. Vehicle-based measures constitute lane changing, steering wheel control, pressure on the pedals etc. Behavioral measures rely on camera inputs to observe the head posture, eye blinking, eye closure and yawning. Physiological measures make use of vital recordings such as the electrocardiogram (ECG), electroencephalogram (EEG) etc. It has been suggested that a system which can fuse the data obtained from multiple sensors (hybrid measures) is best suited for accurate detection of drowsy drivers.

It has also been observed that drowsy drivers juxtapose the usage of brake and accelerator pedals which often leads to undesirous situations on the road. A safety system that avoids unintended acceleration of the vehicle resulting from pedal mix-up has been demonstrated in [16]. A neural network-based method for analyzing the nature of car crashes has been proposed in [17]. A system for modernizing the management of bulk goods transportation and logistics was proposed in [18]. A salient feature of the above system is the use of RFID technology to keep an eye on the movement of delivery trucks.

Though many vehicle safety systems have been proposed in the existing literature, they do not cater to the needs of heavy vehicles such as lorries and trucks. It is well-known that heavy vehicles are often used in the transportation of goods, petroleum products, dairy products, cargo and other commodities. 
Hence, in the present work, we propose a complete ecosystem for the smooth and safe operation of such vehicles by adding many novel features and modules. We have taken care of the overall safety by including different sensors such as touch sensor for emergency, ultrasonic sensor for over height indication, tilt sensor for accident detection, GPS for location identification and RFID to take care of entry and exit of vehicle in restricted areas. However, we have not dealt with drowsiness detection.

Additionally, most of the available designs are based on Raspberry Pi or ARM controller. However, we have used NodeMCU-based approach as our design is simple and does not need any complex signal/image processing algorithms. Similarly, we have used the Message Queuing Telemetry Transport (MQTT) protocol for gathering the sensor data on to the cloud instead of the Hyper Text Transfer protocol (HTTP). It is widely-known that MQTT is well-suited for low-power applications such as IoT [19].

\section{PROPOSED METHOD}

The proposed safety system for heavy vehicles makes use of three NodeMCU units and suitable peripherals for carrying out various functions. The important hardware elements used in the proposed system and their functionality are listed below: -

- ESP8266 system-on-chip for inbuilt Wi-Fi connectivity. It is a part of the NodeMCU.

- A GPS receiver to obtain the location of the vehicle.

- An ultrasonic sensor to detect underpasses that are too short for the vehicle to enter i.e., for over-height indication. A buzzer beeps whenever such underpasses are detected, well-ahead of the entry point, so that the driver can slow down the vehicle.

- A touch sensor-based emergency button.

- A tilt sensor to detect the occurrence of accidents.

- An RFID tag and reader to detect the entry of unauthorized vehicles into restricted areas.

- A DC motor to represent the vehicle ignition.

- $\quad \mathrm{MQ2}$ gas sensor to detect alcohol activity.

\subsection{Hardware Description}

The hardware block diagram of the proposed system is shown in Figure 1. It can be observed that three Node MCU controllers are used to realize the design. At the heart of the system, is the Node MCU2 which forms the central component for accident detection and drunken driving. In both these cases, the vehicle can be stopped by turning off the ignition (DC motor).

Node MCU3 takes care of the over-height indication process using an ultrasonic sensor and a sound buzzer. It has to be noted that Node MCU3 does not have any control over the vehicle's ignition and cannot start or stop the vehicle. Only the driver is alerted using a sound from the buzzer about approaching underpasses whose entrance is shorter than the vehicle's height.

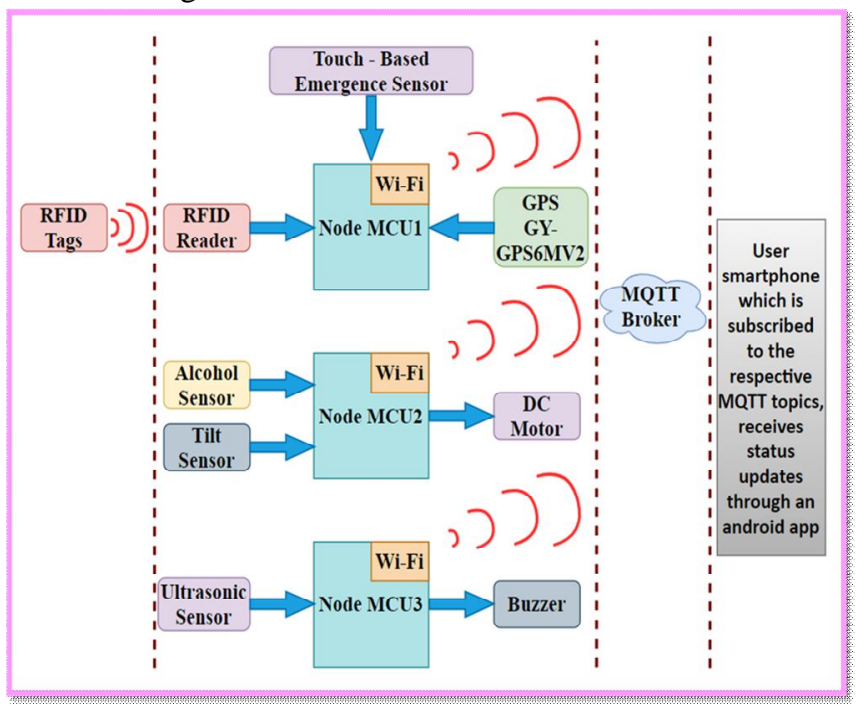

Figure 1: Hardware block diagram of the proposed vehicle safety system.

Similarly, Node MCU1 is interfaced in this prototype to demonstrate the functions such as RFID sensing, gathering location information of the vehicle using GPS as a sensor and emergency sensing using a touch sensor. It can be seen from Figure 1 that the RFID reader is connected to the Node MCU1. However, in practice, the vehicle just contains the RFID tags and the RFID readers are placed at the security gates of the factory premises. The arrangement shown in Figure 1 is only for demonstrating the proposed prototype. The RFID reader interrupts the microcontroller when a RFID tag comes within its range. Similar to Node MCU3, Node MCU1 too has no control on the vehicle's ignition. The GPS sensor is used to provide periodic location updates to the MQTT broker at regular intervals. It has to be noted that this implementation is different from many existing systems where the GPS information can be obtained only after pinging the microcontroller using an SMS or other methods.

All the three NodeMCU controllers operate in parallel. We have removed all potential dependencies between the individual controllers through careful designing. For example, the GPS module is made to send periodic location updates to the cloud instead of the regular on-demand location updates used in most designs. Similarly, the touch-based emergency sensor is de-linked from the vehicle ignition and is used only as an indicator to the control room using the cloud-based platform. The NodeMCU3 takes care of the over-height indication but does not switch off the ignition.

\subsection{Process Flow or Control Path of the Proposed System}

The high-level representation of the proposed system 
operation is depicted in Figure 2. As most of the modules operate independently, they are showcased parallel to each other, thereby indicating that there are no control dependencies between them.

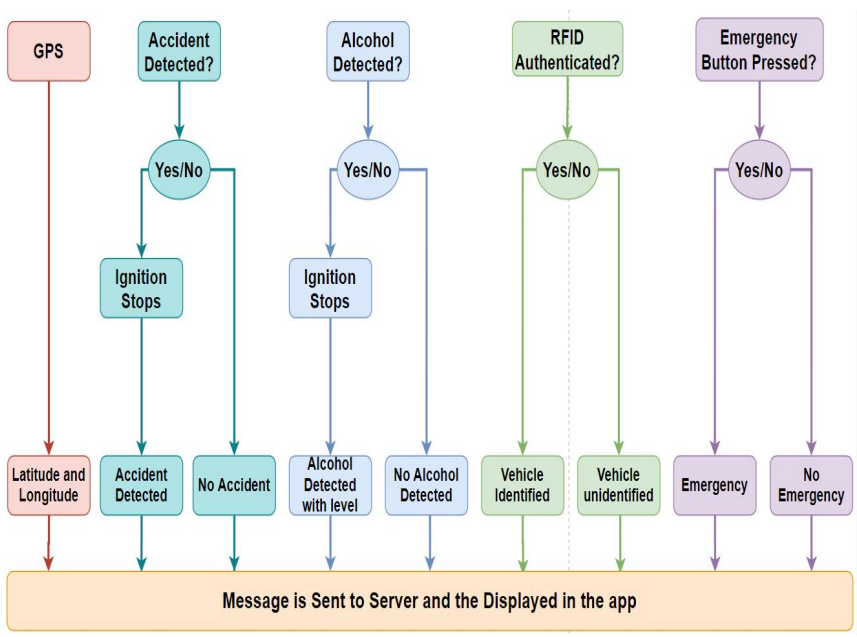

Figure 2: Flow diagram for operation of the proposed system

It can be observed that the GPS module operates on its own and updates the location information of the vehicle to the MQTT broker from time to time from where the user can get the data on his/her smartphone. All other modules update their current status to the MQTT broker after checking the respective parameters. The user can monitor the status of all these sensors from a remote location by subscribing to the relevant MQTT topics in the smartphone. The reasons for preferring MQTT protocol over HTTP are explained below.

A block diagram representing the operation of the MQTT protocol is shown in Figure 3. At the heart of MQTT protocol is the MQTT broker (server), which handles dispatch of messages between senders and receivers (clients). Each client that publishes a message to the broker includes a topic into that message. Clients which want to receive a message need to subscribe to a particular topic. The broker delivers the message with the matching topic to the respective clients. Client devices communicate on topics of interest with the help of the MQTT broker and hence need not know the identity of each other.

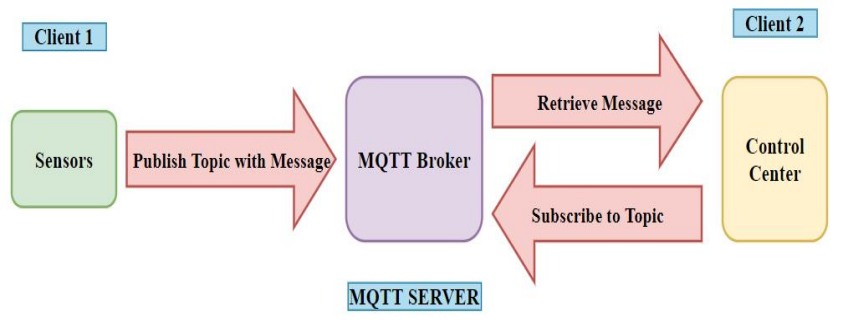

Figure 3: Essential elements of the MQTT protocol

The differences between MQTT and HTTP are listed out in Table 1. MQTT is preferable for low-power and low-bandwidth applications and in situations where quick response times, high throughput and intermittent connectivity are needed.
Table 1: Differences between MQTT and HTTP

\begin{tabular}{|l|l|}
\hline \multicolumn{1}{|c|}{$\begin{array}{c}\text { Message Queue Telemetry } \\
\text { Transport (MQTT) }\end{array}$} & \multicolumn{1}{c|}{$\begin{array}{c}\text { Hyper Text Transfer } \\
\text { Protocol (HTTP) }\end{array}$} \\
\hline $\begin{array}{l}\text { It is event-driven and based on } \\
\text { publish-subscribe } \\
\text { architecture. }\end{array}$ & $\begin{array}{l}\text { It is document-centric and } \\
\text { based on request-response } \\
\text { architecture. }\end{array}$ \\
\hline $\begin{array}{l}\text { Message format is binary. } \\
\text { Short headers and short packet } \\
\text { sizes. MQTT has pretty short } \\
\text { specifications and is } \\
\text { lightweight. }\end{array}$ & $\begin{array}{l}\text { Message format is ASCII. } \\
\text { Lengthy headers and } \\
\text { messages. HTTP has } \\
\text { detailed specifications and } \\
\text { is more optimized for } \\
\text { client-server interactions. }\end{array}$ \\
\hline $\begin{array}{l}\text { It has many features to } \\
\text { guarantee the delivery of } \\
\text { messages. }\end{array}$ & $\begin{array}{l}\text { Not many features to } \\
\text { guarantee message delivery. }\end{array}$ \\
\hline
\end{tabular}

Overall, it can be concluded that MQTT is suitable for IoT sensing applications where small amounts of sensor data has to be updated to the cloud using resource-constrained devices [20], [21].

\subsection{Description of Key Hardware Components}

Table 2 provides the specifications of the key hardware components used for the prototype. Part numbers, a small image and a brief description of each hardware component are given.

Table 2: Key Hardware Components

\begin{tabular}{|c|c|c|c|}
\hline \multirow{2}{*}{$\begin{array}{l}\text { Hardwar } \\
\text { e Module }\end{array}$} & \multicolumn{3}{|c|}{ Specifications } \\
\hline & Description & $\begin{array}{c}\text { Model } \\
\text { Number }\end{array}$ & Image \\
\hline $\begin{array}{l}\text { Ultrasonic } \\
\text { sensor } \\
{[22]}\end{array}$ & $\begin{array}{l}\text { Operating frequency } \\
40 \mathrm{kHz} \text {, operating } \\
\text { voltage } 5 \mathrm{~V} \text {. Obstacle } \\
\text { detection range } 2 \mathrm{~cm}- \\
400 \mathrm{~cm} \text { (theoretical), } 2 \\
\mathrm{~cm}-80 \mathrm{~cm} \text { (practical), } \\
\text { non-contact sensing. } \\
\text { Range accuracy } 3 \mathrm{~mm} \text {. } \\
\text { Module has ultrasonic } \\
\text { transmitter, receiver } \\
\text { and control unit. Has } 4 \\
\text { pins }\left(\mathrm{V}_{\mathrm{CC}} \text {, Ground, }\right. \\
\text { Trigger Pulse and } \\
\text { Echo pulse). } \\
\text { Measuring angle } \\
\text { covered } 15^{\circ} \text {. }\end{array}$ & HC-SR04 & \\
\hline $\begin{array}{c}\text { Gas sensor } \\
{[23]}\end{array}$ & $\begin{array}{l}\text { Operating voltage } 5 \mathrm{~V} \text {, } \\
\text { power consumption } \\
800 \mathrm{~mW} \text {. Can detect } \\
\text { LPG, methane, smoke, } \\
\text { alcohol, propane etc., } \\
\text { with concentrations } \\
\text { between } 200-10^{4} \\
\text { ppm. Has } 4 \text { pins. } \\
\text { Preheat duration } 20 \\
\text { seconds. }\end{array}$ & MQ2 & \\
\hline
\end{tabular}




\begin{tabular}{|c|c|c|c|}
\hline $\begin{array}{c}\text { RFID Tag } \\
\text { and RFID } \\
\text { Reader } \\
{[24]}\end{array}$ & $\begin{array}{l}\text { Operating voltage } 2.5 \text { - } \\
3.3 \mathrm{~V} \text {. Read range } 5 \\
\mathrm{~cm} \text {. Operating } \\
\text { frequency: } 13.56 \mathrm{MHz} \text {, } \\
\text { peak data transfer rate: } \\
\text { 10Mbps. Consists of } 8 \\
\text { pins. Can be interfaced } \\
\text { to host using } \\
\text { SPI/I2C/UART } \\
\text { standards. }\end{array}$ & RC522 & 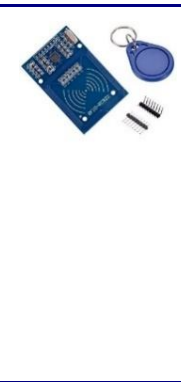 \\
\hline $\begin{array}{c}\text { GPS } \\
\text { module } \\
{[25]}\end{array}$ & $\begin{array}{l}50 \text { channels, GPS L1 } \\
\text { band (1.575GHz), } 1 \\
\text { Hz position update } \\
\text { rate (peak } 5 \mathrm{~Hz}), 3-5 \mathrm{~V} \\
\text { power supply, } \\
\text { sensitivity of -161 } \\
\text { dBm, configurable } \\
\text { baud rate. Has } 4 \text { pins } \\
\text { (VC, Ground, TxD, } \\
\text { RxD). An LED on the } \\
\text { module indicates the } \\
\text { device's status. } \\
\text { Supports many } \\
\text { protocols including } \\
\text { NMEA. }\end{array}$ & NEO-6M & \\
\hline
\end{tabular}

\section{PROTOTYPE DESIGN METHODOLOGY}

This section explains the methodology followed for designing a working prototype of the proposed system.

\subsection{Hardware Interfacing}

The detailed port level connections used for connecting the various hardware components described in the previous section for designing the prototype are given in Table 3.

Table 3: NodeMCU port numbers to connect peripherals

\begin{tabular}{|l|l|l|}
\hline $\begin{array}{l}\text { Hardware } \\
\text { components }\end{array}$ & Port numbers & $\begin{array}{l}\text { NodeMCU } \\
\text { number }\end{array}$ \\
\hline $\begin{array}{l}\text { Emergency touch } \\
\text { button }\end{array}$ & D0 & 1 \\
GPS & D3 & 1 \\
RFID scanner & D2, D5, D7, D6, D1 & 1 \\
Alcohol sensor & A0 & 2 \\
DC motor & D7 & 2 \\
Tilt sensor & D1 & 2 \\
Ultrasonic sensor & D1, D2 & 3 \\
Buzzer & D4 & 3 \\
\hline
\end{tabular}

\subsection{Software Implementation}

An MQTT broker was set up for gathering the data from various sensors on to the cloud. A suitable client ID, server name and port number has to be assigned as shown in Figure 4 in order to set up the broker. This allows the user to monitor the status of all the modules on the vehicle safety system using a smartphone with the corresponding android app.

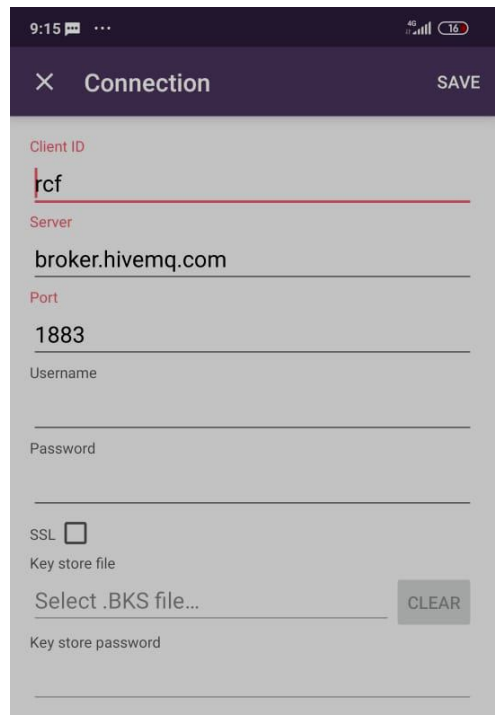

Figure 4: Setting up the MQTT broker

\section{RESULTS AND DISCUSSION}

Figure 5 shows the top view of the hardware prototype. The hardware components such as the ultrasonic sensor, buzzer, RFID reader and tags, DC motor, tilt sensor and alcohol sensor can be seen in the picture. This system increases efficiency of transportation of goods to their respective destination in a safe and secure way.

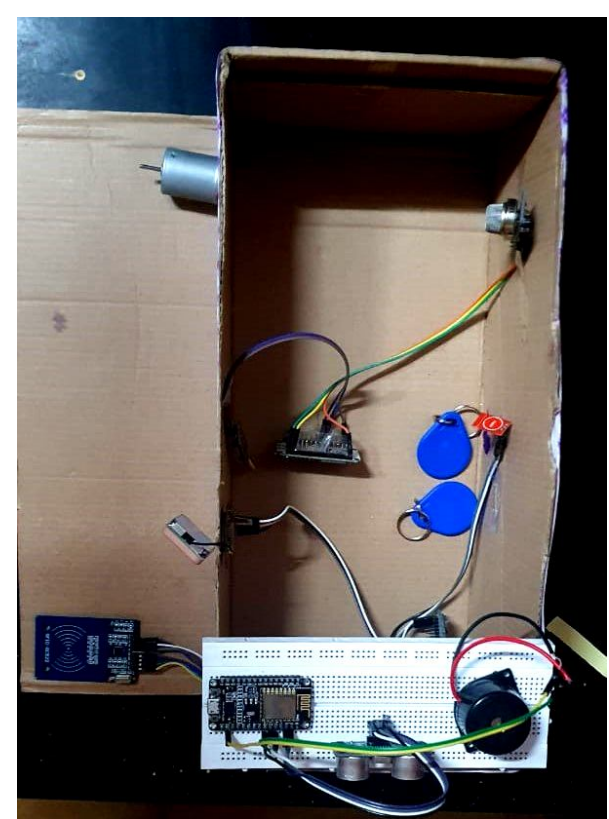

Figure 5: Top view of the hardware prototype

\subsection{GPS functionality}

The GPS System helps to track the site of the truck to get complete information of the location of the vehicle. Figure 6 shows the current coordinates of the vehicle received in the smartphone application. These coordinates can be placed in Google maps to identify the vehicle's location. For fleet 
services, authorities can monitor the location of all their vehicles and the duration for which a particular vehicle stays in a given location.

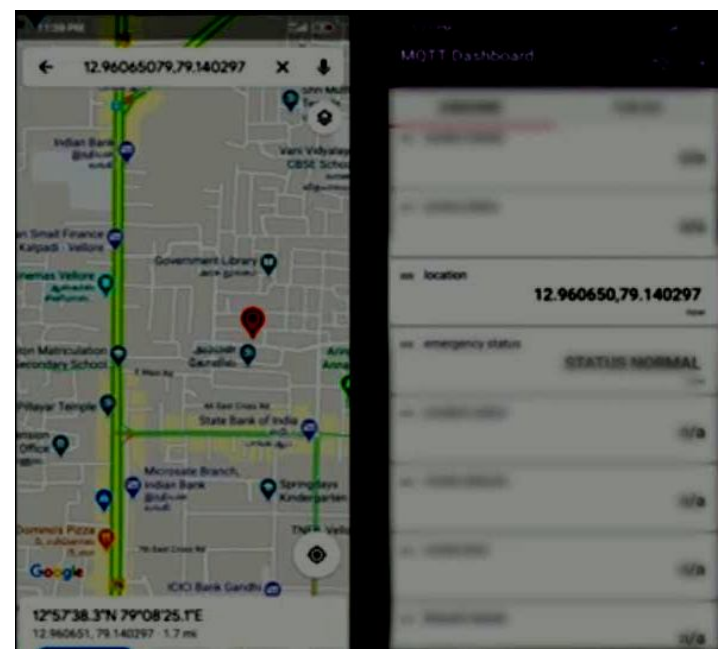

Figure 6: Location information obtained from GPS sensor

\subsection{RFID functionality}

The vehicle is provided with a RFID tag which will be scanned during its entry and exit into or out of a particular location. Figure 7 shows the output obtained from the RFID reader when a vehicle with a valid tag is scanned. In a similar way, figure 8 shows the output when an unauthorized vehicle comes in the vicinity of the RFID scanner. This system of radio identification of vehicles helps the authorities to keep an eye on the movement of vehicles within the factory premises and helps to keep track of the consignments.

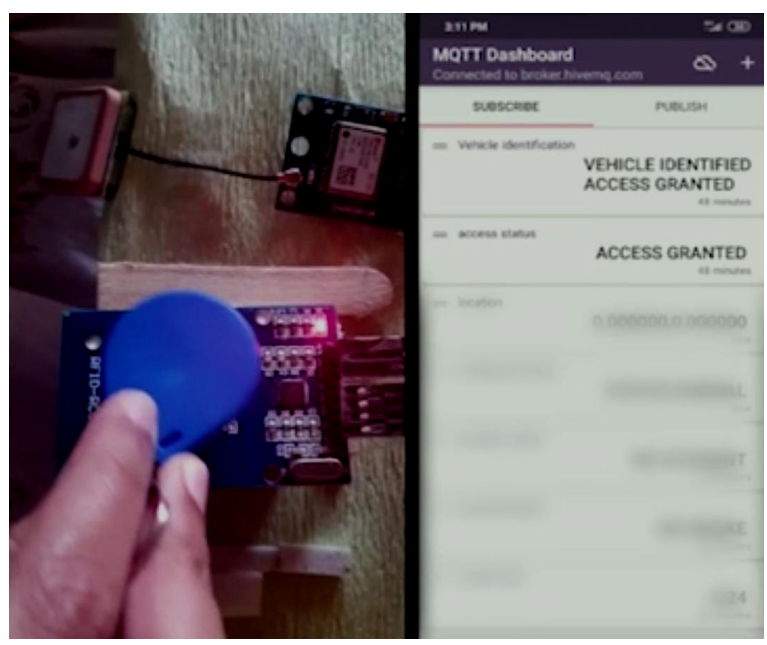

Figure 7: RFID tag authorized and access granted

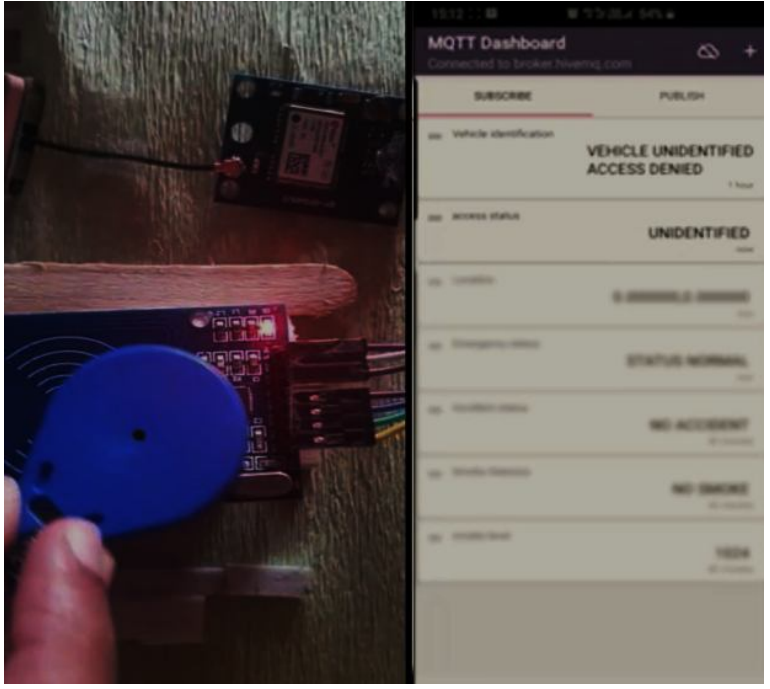

Figure 8: RFID tag unauthorized and access denied

\subsection{Emergency Button}

A touch-based emergency button is provided near the driver's seat so that the driver can manually access the touch sensor to indicate an emergency. This increases the reliability of the system and avoids over-reliance on automation as there may be times at which the sensors may fail to detect an emergency. Figure 9 shows the normal situation when the touch sensor is not accessed. Figure 10 indicates an emergency when the driver touches the sensor.

\subsection{Alcohol Activity Detection}

Figure 11 shows the message received in the app when the alcohol content is sensed using the MQ2 sensor. This helps in the prevention of drunk and drive cases.

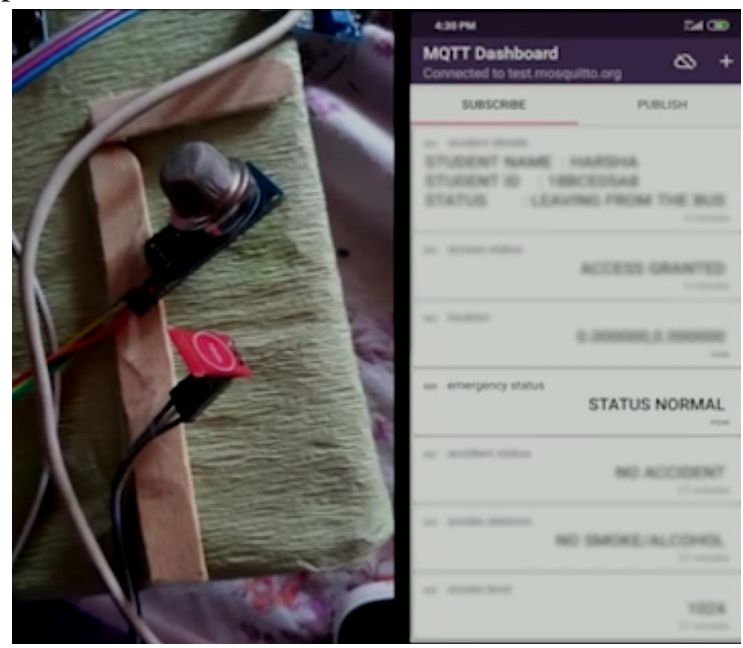

Figure 9: No emergency as sensor is untouched 


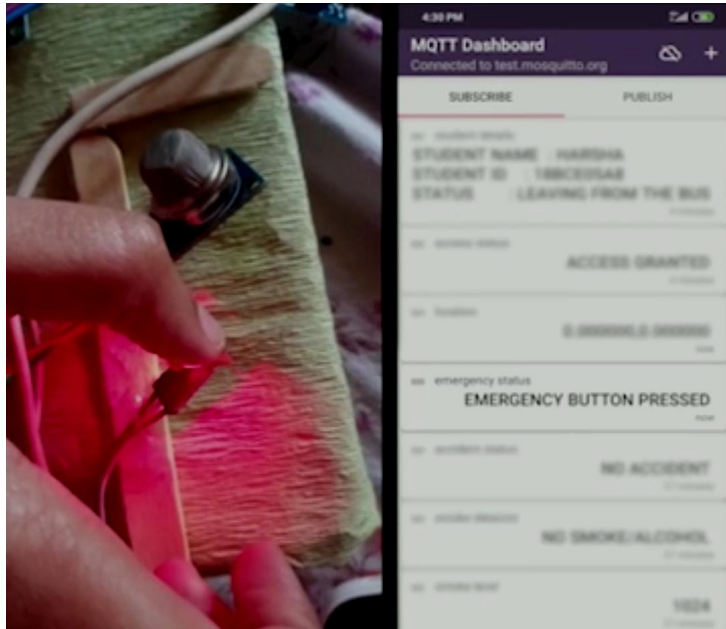

Figure 10: Emergency situation as sensor is touched

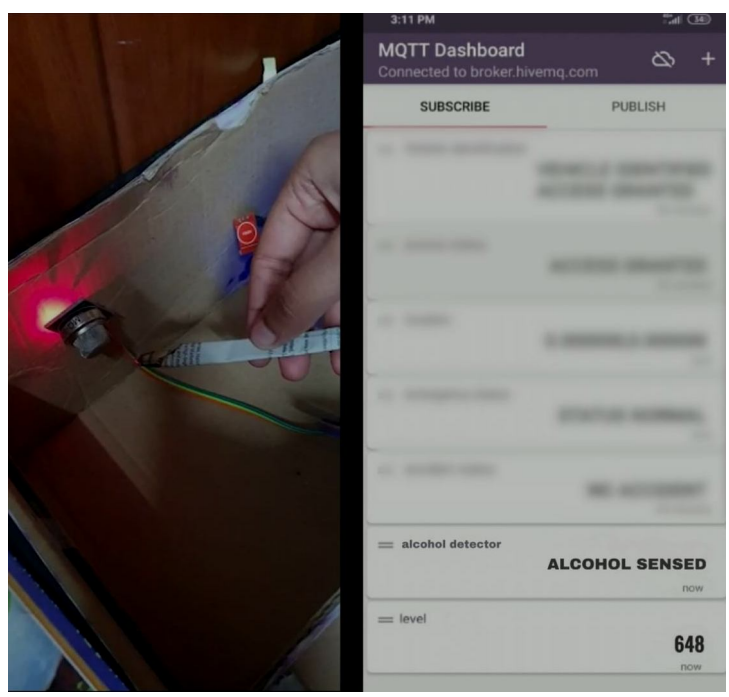

Figure 11: Alcohol activity sensed

\subsection{Accident Detection}

Most of the time, the lives of the travellers are lost due to the delay in sending the rescue team for offering appropriate help.

Figure 12 shows the output when the tilt sensor is in normal position, thereby indicating that there is no change in the angular orientation of the vehicle. Figure 13, on the other hand, shows that the tilt sensor is held upside down and hence detects the occurrence of accident. The accident is being detected with the help of the tilt sensor. When the accident takes place, the message is immediately sent to the app which will be available with the responsible authorities so that suitable help can be provided to the vehicle and the driver.

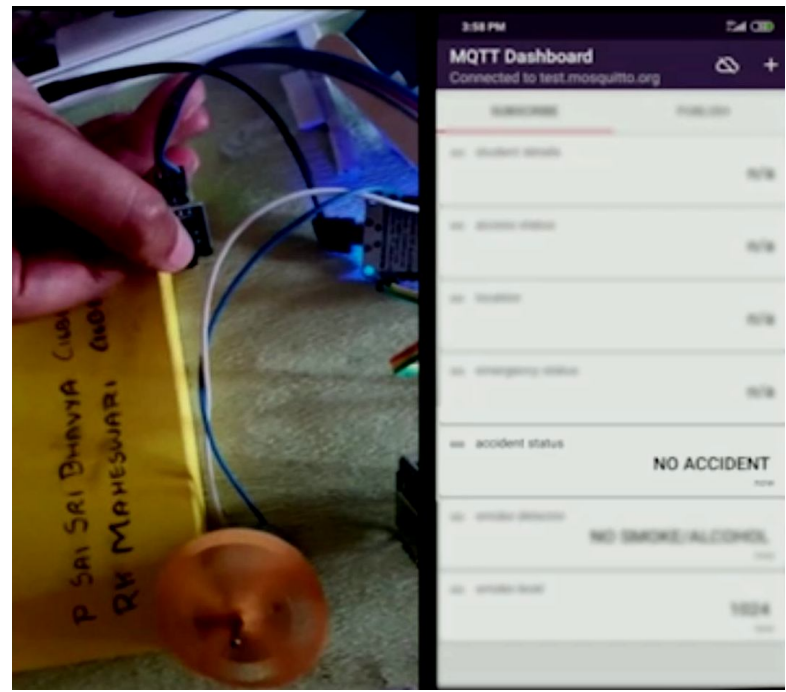

Figure 12: No accident - Motor (Ignition) is on

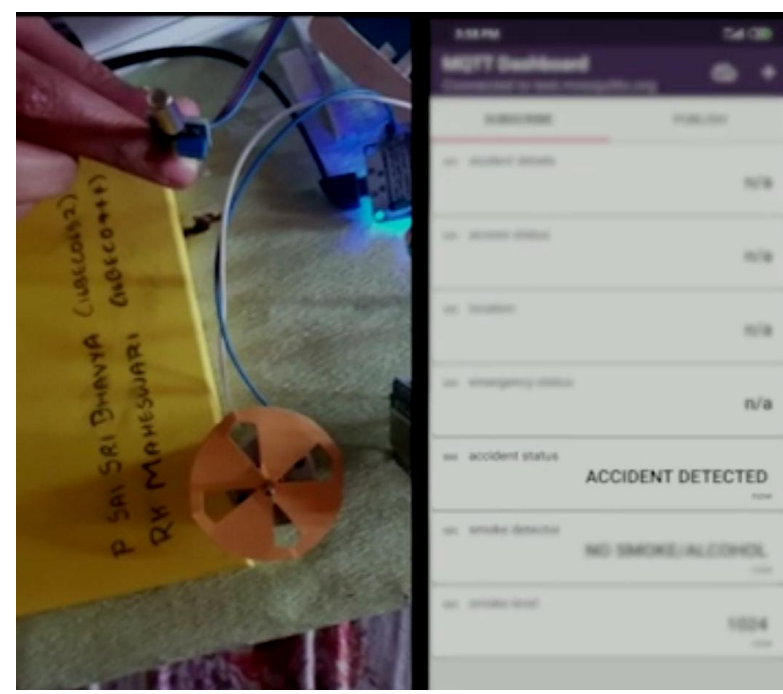

Figure 13: Accident occurred - Motor (Ignition) turns off

\subsection{Over-height Indication}

Figure 14 shows the ultrasonic sensor and buzzer assembly for over-height indication. From a design perspective, this assembly has to be mounted on the topmost point of the vehicle. Ideally, there should be ultrasonic sensors throughout the front panel of the vehicle in order to identify the underpasses which are smaller than it. However, since this prototype is just to demonstrate the concept, we have used only one ultrasonic sensor. Whenever there is an obstacle in the line of sight of the ultrasonic sensor, the buzzer starts a sound alarm. This way, the driver gets alerted and can slow down the vehicle. Such a system is beneficial during foggy conditions or when drowsy/drunk drivers fail to visually judge the height of tunnels under the influence of sleep or alcohol. 


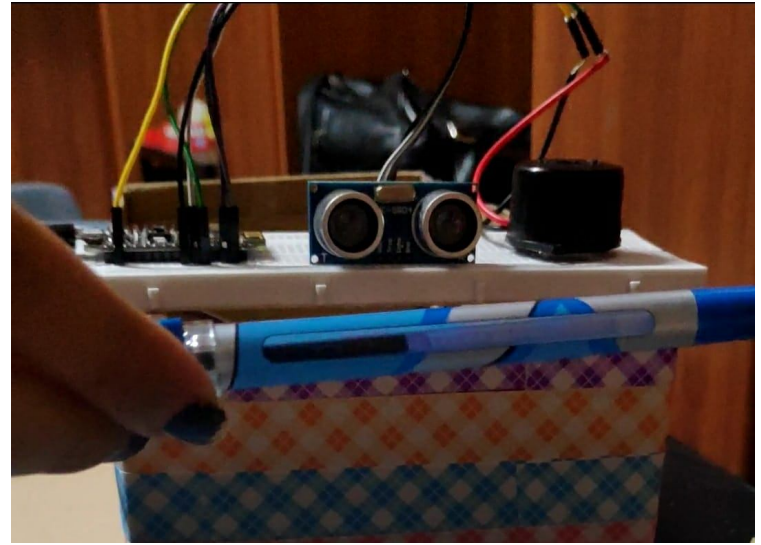

Figure 14: Over-height indicator assembly

It is therefore seen that all the modules in the proposed system have been designed and tested successfully.

\section{CONCLUSION AND FUTURE SCOPE}

The proposed heavy vehicle safety system using NodeMCU and suitable peripherals has been successfully designed, prototyped and tested for functionality. It meets the initial design specifications and operates as per the desired functionality. The system is reliable, user friendly and quite inexpensive. It provides a complete ecosystem for monitoring the safety and security of heavy vehicles.

In future, a speed locking mechanism can be built into the system similar to the one designed in [9]. The system could also be upgraded to act as a vehicle black box to carry out post-crash analysis [26], [27] after adding additional sensors. In addition, a microphone assembly could also be interfaced to the automobile black box to record driver's conversations similar to those available in airplane cockpits.

\section{REFERENCES}

[1] D. Dipak. Over 1.51 lakh died in road accidents last year; UP tops among states | India News - Times of India, The Times of India, New Delhi, Nov. 16, 2019.

[2] Ministry of Road Transport and Highways. Road_Accidednts.pdf. Accessed: Apr. 28, 2020. [Online]. Available: https://morth.nic.in/sites/default/files/Road_Accidednts. pdf, 2019.

[3] A. Celesti, A. Galletta, L. Carnevale, M. Fazio, A. Ĺay-Ekuakille, and M. Villari. An IoT Cloud System for Traffic Monitoring and Vehicular Accidents Prevention Based on Mobile Sensor Data Processing, IEEE Sens. J., vol. 18, no. 12, pp. 4795-4802, Jun. 2018, doi: 10.1109/JSEN.2017.2777786.

[4] M. Vyas Viral, V. Choksi, and M. B. Potdar. Car Safety System Enhancements using the Internet of Things (IoT), Int. Res. J. Eng. Technol. IRJET Vol.-04 Issue-12, 2017.
[5] Z. Xiaorong, F. Honghui, F. Zhongjun, C. Dan, G. Zixia, and F. Hanyu. The Drunk Driving Automatic Detection System Based on Internet of Things, Int. J. Control Autom., vol. 9, no. 2, pp. 297-306. https://doi.org/10.14257/ijca.2016.9.2.28

[6] K. Sandeep, P. Ravikumar, and S. Ranjith. Novel Drunken Driving Detection and Prevention Models Using Internet of Things, in 2017 International Conference on Recent Trends in Electrical, Electronics and Computing Technologies (ICRTEECT), Jul. 2017, pp. 145-149, doi: 10.1109/ICRTEECT.2017.38.

[7] A. Patwari, G. Kavya, and R. Meghana. Fleet Monitoring System using GSM, GPS and Web Server with Drunken Driving Alerts, Int. J. Innov. Technol. Explor. Eng., vol. 9, no. 3, pp. 2080-2084, Jan. 2020, doi: 10.35940/ijitee.C8924.019320.

[8] H. Yadav, M. Varshney, H. Gupta, and H. C. Pal. Design and Implementation of Advance Accident Prevention System for Reducing the Cause of Death, Int. J. Radio Freq. Des., vol. 5, no. 1, pp. 1-5, Jul. 2019.

[9] G. Shanmugasundaram, A. Anil, S. Deepak, and F. Ahmed. Smart accident alert and toll pay system, in 2017 Fourth International Conference on Signal Processing, Communication and Networking (ICSCN), Mar. 2017, pp. 1-5, doi: 10.1109/ICSCN.2017.8085691.

[10] Ananya M B, Nisarga N C, Vardhini V Hebballi, S B Rudraswamy. Semi-Automted IoT Vehicles, in 2019 IEEE International Conference on Electronics, Computing and Communication Technologies (CONECCT), Jul. 2019, pp. 1-8, doi: 10.1109/CONECCT47791.2019.9012866.

[11] P. K. Binu, K. J. Sredhey, and R. S. Anuvind. An Iot Based Safety and Security Mechanism for Passenger Vehicles, in 2019 2nd International Conference on Intelligent Computing, Instrumentation and Control Technologies (ICICICT), Jul. 2019, vol. 1, pp. 1502-1506, doi: 10.1109/ICICICT46008.2019.8993177.

[12] F. Jabeen, S. R. Rupanagudi, and V. G. Bhat. IoT based Smart Vehicle Ignition and Monitoring System, in 2019 International Conference on Advances in Computing, Communication and Control (ICAC3), Dec. 2019, pp. 1-7, doi: 10.1109/ICAC347590.2019.9036809.

[13] J. James and S. V. Suryakala, Advanced vehicle security control and accident alert system, Int. J. Eng. Technol., vol. 7, no. 2.8, pp. 404-408, Mar. 2018, doi: 10.14419/ijet.v7i2.8.10679.

[14] I. F. Rahmad, E. B. Nababan, L. Tanti, B. Triandi, E. Ekadiansyah, and V. A. Fragastia. Application of the Alcohol Sensor MQ-303A to Detect Alcohol Levels on Car Driver, in 2019 7th International Conference on Cyber and IT Service Management (CITSM), Nov. 2019, vol. 7, pp. 1-5, doi: 10.1109/CITSM47753.2019.8965395.

[15] A. Sahayadhas, K. Sundaraj, and M. Murugappan. Detecting Driver Drowsiness Based on Sensors: A 
Review, Sensors, vol. 12, no. 12, pp. 16937-16953, Dec. 2012, doi: 10.3390/s121216937.

[16] S. Priyanka, G. Hemalatha, and C. Saranya. Sudden unintended acceleration avoidance and drowsiness detector for automobile accidents prevention, in 2017 Third International Conference on Science Technology Engineering Management (ICONSTEM), Mar. 2017, pp. 964-967, doi: 10.1109/ICONSTEM.2017.8261346.

[17] S. Mothe. A Model for Assessing the Nature of Car Crashes using Convolutional Neural Networks, Int. J. Emerg. Trends Eng. Res., vol. 8, no. 3, pp. 859-863, Mar. 2020, doi: 10.30534/ijeter/2020/41832020.

[18] O. O. Gorshkova. Modernization of Transport Management System upon Conveyance of Bulk Goods, Int. J. Emerg. Trends Eng. Res., vol. 8, no. 3, pp. 659-662, Mar. 2020, doi: 10.30534/ijeter/2020/08832020.

[19] N. Naik. Choice of effective messaging protocols for IoT systems: MQTT, CoAP, AMQP and HTTP, in 2017 IEEE International Systems Engineering Symposium (ISSE), Oct. 2017, pp. 1-7, doi: 10.1109/SysEng.2017.8088251.

[20] S. Satya Shankar. Use MQTT Protocol In Smartphones And Raspberry Pi, Electronics For You, Feb. 24, 2020.

[21] M. Serozhenko. MQTT vs. HTTP: which one is the best for IoT?, Medium, Mar. 20, 2017. https://medium.com/mqtt-buddy/mqtt-vs-http-which-on e-is-the-best-for-iot-c868169b3105 (accessed Apr. 27, 2020).

[22] HC-SR04 Ultrasonic Sensor: Working, Pin Diagram, Description \& Datasheet.

https://components101.com/ultrasonic-sensor-workingpinout-datasheet (accessed Apr. 27, 2020).

[23] MQ2 Gas Sensor Pinout, Features, Equivalents \& Datasheet. https://components101.com/mq2-gas-sensor (accessed Apr. 27, 2020).

[24] RC522 RFID Module Pinout, Features, Specs \& How to Use It.

https://components101.com/wireless/rc522-rfid-module (accessed Apr. 27, 2020).

[25] In-Depth: Interface ublox NEO-6M GPS Module with Arduino, Last Minute Engineers, Nov. 11, 2018. https://lastminuteengineers.com/neo6m-gps-arduino-tut orial/ (accessed Apr. 27, 2020).

[26] V. Pagadala, S. Rani, and B. K. Priya. Design and Implementation of the Prevention and Analysis of the Accident for Automobiles, in 2018 International Conference on Advances in Computing, Communications and Informatics (ICACCI), Sep. 2018, pp. 2283-2289, doi: 10.1109/ICACCI.2018.8554473.

[27] M. J. Prasad, S. Arundathi, N. Anil, Harshikha, and B. S. Kariyappa. Automobile black box system for accident analysis, in 2014 International Conference on Advances in Electronics Computers and Communications, Oct. 2014, pp. 1-5, doi: 10.1109/ICAECC.2014.7002430. 